\title{
Treatment of Idiopathic Granulomatous Mastitis: Combined Intralesional Steroid Injection with Topical Steroid Administration vs. Systemic (Oral) Steroid
}

\section{Osman toktas ( $\nabla$ osmantoktas@windowslive.com )}

Yuzuncu Yil University: Van Yuzuncu Yil Universitesi https://orcid.org/0000-0003-1277-1358

\section{Nursen Toprak}

Yuzuncu Yil University Faculty of Medicine: Yuzuncu Yil Universitesi Tip Fakultesi

\section{Sadi Elasan}

Yuzuncu Yil University Faculty of Medicine: Yuzuncu Yil Universitesi Tip Fakultesi

\section{Iskan Calli}

Yuzuncu Yil University Faculty of Medicine: Yuzuncu Yil Universitesi Tip Fakultesi

\section{Serhat Binici}

Yuzuncu Yil University Faculty of Medicine: Yuzuncu Yil Universitesi Tip Fakultesi

\section{Research}

Keywords: Idiopathic granulomatous mastitis, mastitis, steroid injection, topical steroid, systemic steroid

Posted Date: November 11th, 2021

DOI: https://doi.org/10.21203/rs.3.rs-1032751/v1

License: @) (i) This work is licensed under a Creative Commons Attribution 4.0 International License. Read Full License 


\section{Abstract}

\section{Background}

Idiopathic granulomatous mastitis (IGM)is characterized by non-caseating granuloma and microabscess formation limited to mammary gland lobules. It is a form of chronic mastitis of unknown pathogenesis. In this study, we aimed to compare the effectiveness of intralesional steroid injection with topical steroids versus systemic steroid therapy in the treatment of IGM.

\section{Methods}

Between June 2017 and December 2020, the patients were collected and assessed. IGM was diagnosed histopathologically by tru-cut biopsy in patients with breast mass, pain, and erythema with suspicion of IGM. Included in the study were one hundred and eleven patients who were diagnosed with IGMand who were treated with local or oral administration of corticosteroids, with at least 6 months of follow-up. The patients were divided into 2 groups: a local corticosteroid treatment group (LC, group 1, $\mathrm{n}=57$ ); and a peroral corticosteroid treatment group (OC, group 2 , $n=54)$. Demographic characteristics, treatment responses, recurrence rates, side effects of the steroid, and the need for surgery were compared.

\section{Results}

While the rate of smoking was $12.3 \%$ in the LC group, was $20.4 \%$ in the OC group. There was no history of oral contraceptive use in either group of patients. Previous steroid use was significantly lower in the LC group (10.5\%) compared to the OC group (55.6\%) ( $p=0.001)$. Previous antibiotic use was significantly lower in the LC group (75.4\%) compared to the OC group (100\%) ( $p=0.001)$. When complete and partial responders were grouped as "responders," $96.5 \%$ in the LC group and $75.9 \%$ in the OC group responded after the first course of treatment $(p=0.001) .98 .2 \%$ in the LC group and $87.0 \%$ in the OC group were complete responders after the third course of treatment $(p=0.003)$. While $7 \%$ of the patients in the LC group had recurrence, $37 \%$ in the OC group had recurrence $(p=0.001)$. Steroid-related side effects were lower in the LC group (no) compared to the OC group $(11.1 \%)(p=0.010)$. Surgery was performed in $3.5 \%$ of the LC group, while surgery was performed in $57.3 \%$ of the OC group $(p=0.001)$.

\section{Conclusion}

Steroid injection is an IGM treatment with proven efficacy, short response time, low recurrence rate, and lower need surgery. The treatment is easy to administer and minimizes the risk of systemic side effects. Even in clinically severe cases, we believe that steroid injection might be used as monotherapy, or in combination with other therapies, to improve treatment efficacy and create a transition to less aggressive treatments.

\section{Introduction}

Idiopathic granulomatous mastitis (IGM)is characterized by non-caseating granuloma and microabscess formation limited to mammary gland lobules. It is a form of benign chronic mastitis of unknown pathogenesis. Kessler and Wolloch first described this disease in 1972 [1, 2]. IGM is usually observed as a persistent or recurrent tender and inflammatory mass in the breast in premenopausal women with a history of breastfeeding. Although the etiology of IGM is still not well known, many different developmental mechanisms have been suggested in the literature. While the autoimmune response is the most accepted theory, infection and hormonal disruption are considered the three leading hypotheses [3]. Because IGM is clinically similar to breast cancer and inflammatory breast diseases, IGM needs to be differentiated from breast cancer and granulomatous inflammation or autoimmune diseases such as tuberculosis, 
sarcoidosis, fungal/parasitic infection, and Wegener's granulomatosis [4]. Epidemiologically, IGM is rare and accounts for approximately $0.44-1.6 \%$ of breast biopsy specimens. IGM is more common in Asian and Spanish women, and it is mostly seen in childbearing age [5]. Misdiagnosis of IGM is common, especially in the beginning, and it can turn into a frustrating long-term illness for the patient and doctor. There is still no consensus on the treatment of IGM. Treatment includes many options, such as surgical resection, steroids, methotrexate, bromocriptine, colchicine, immunosuppressive agents, antibiotics and even follow-up observation [6]. Currently, treatment mostly depends on the experience of the clinic or surgeon and the severity of the patient's disease [7].

In this study, we compared the efficacy of local steroid therapy that is combined intralesional steroid injection with topical steroid administration versus systemic steroid therapy in the treatment of IGM.

\section{Materials And Methods}

The data collected between June 2017 and December 2020, was analyzed retrospectively. Patients who presented with breast mass, breast pain, mastitis, and suspected idiopathic granulomatous mastitis underwent core needle abscess drainage and incisional biopsy, or excisional biopsy, according to their clinical presentation. The diagnosis of IGM was made histopathologically. Infectious granulomatous mastitis agents were excluded with Gram, Periodic AcidSchiff (PAS), Ziehl-Neelsen (EZN) and Giemsa Stains in the histopathological diagnosis of each patient. Included in the study were patients diagnosed with IGM who were treated with oral or local administration of corticosteroids, with at least 6 months of follow-up. Patients with recurrent disease or a contraindication for the use of corticosteroids, patient has breast abscess, and patients who were pregnant or lactating were excluded from the study. Extracted from patients' files were their age, number of pregnancies, history of smoking, complaint at disease onset, radiologic method preferred for the diagnosis and follow-up [such as breast ultrasound (USG), mammography (MMG) and breast magnetic resonance imaging (MRI) depending on the patients age and clinical status], and the biopsy method used for diagnosis. No specific patient selection was made for the two groups, the patients were selected consecutively. We didn't calculate sample size. The patients were selected consecutively according to the order they applied to the clinic. In our clinic, we have tried to treat almost all patients with IGM with LC for the last 2 years. Most of the patients in the $\mathrm{OC}$ group were patients who were treated before 2 years. No choice was made regarding the extent of the disease between the two groups.

\section{Treatment protocol}

There were 2 patient groups: a local corticosteroid treatment group (LC, group $1, n=57)$ and a peroral corticosteroid treatment group (OC, group 2, $n=54)$. LC protocol was an intralesional triamcinolone acetonide which a medium-tostrong potency corticosteroid (Kenacort-A amp. $40 \mathrm{mg} / \mathrm{ml}$, Deva, Istanbul, Turkey) injection in the lesions once a month, under the USG guided (Figure 1) and topical administration of triamcinolone acetonide $0.1 \%$ pomade (Kenacort-A $\% 0.1,20 \mathrm{gr}$ pomad, Deva, Istanbul, Turkey) on the skin of the affected breast area twice a day on alternate days for one month. We diluted 1 amp of Keacort-A with 3-5 cc saline to the part of the lesion, depending on the size of the lesion. Injection was made at different points at $5 \mathrm{~cm}$ interval in patients with diffuse disease. Injections were made separately. In patients with multiple lesions if groups of lesions were more than $5 \mathrm{~cm}$ apart and if the individual lesions were less than 5 apart, the larger group was injected. OC protocol was administered as methylprednisolone $32 \mathrm{mg} /$ day, with each tablet containing $16 \mathrm{mg}$ methylprednisolone. (Prednol $16 \mathrm{mg}$ tb, Deva, Istanbul, Turkey). Before the treatment, the patient was informed about the treatments. however, 4 patients were excluded from treatment because they could not follow up due to treatment non-compliance. While About $10 \%$ of the LC patients and about half of the OC patients were treated in other centers. After this treatment patients applied to our clinic for biopsy or surgery. LC treatment lasted for 1 month. We diluted 1 amp of the drug into 3-5 cc and injected it into the area where the 
disease was. The patients felt pain in the injection, but this pain was not intolerable. It was done just like a normal injection. Also, we have rescue medications as a methotrexate, but we don't need to use it.

One month later, the response of the patients was evaluated with clinical and radiological ultrasonography in cases who completed their treatment. Patients were classified depending on resolution of the disease clinically and radiologically as "complete response," "partial response," "no response," or "worsening disease." Patients with "complete response" were consigned to follow-up, while patients with "partial response", "no response," and "worsening disease" received a second and if necessary, a third course of treatment with monthly check-ups, until the complete resolution of the disease. If there was no significant improvement in the disease despite the treatments in one patient, we considered this as a persistent disease. If a patient had complete resolution after treatment, after a while mastitis seen again, we defined that the recurrence of the disease. The reason for the long follow-up period in the OC group was these patients were treated in the first years. We started the follow-up period from the time the patients' treatment ended. Information about the course of the disease available treatment options, and their rate of success were given all patients at their initial and follow-up exams. Surgical treatment was performed for patients who no response or worsening disease, and preferred surgery during follow-up. In our clinic, we do not perform surgical treatment on almost any of the patients with IGM. We have been trying to treat it completely with LC in the last 2 years.

The various treatment modalities were low or single box OC, various antibiotics, surgury such as lumpectomy and abscess drainage, and follow-up. Each "course" of OC treatment lasted for 1 month and was taper-off the steroid regimen by reducing the steroid dose. LC treatment was not applied to the group in which OC was used, and vice versa. There is only one patient who underwent lumpectomy in the LC group, and that patient was also a patient who did not respond to treatment. In fact, it had a medical indication. Although we wanted to continue LC treatment, the patient used the choice from surgery. He had used antibiotherapy for a long time and did not provide any benefit. We considered the patient's choice reasonable. No surgery was performed without medical indication. All patients who underwent surgery had medical indications.

\section{Statistical Analysis}

In calculating the sample width of the study, Power (the power of the test) of at least $80 \%$ for each variable and Type 1 error were determined by taking $5 \%$. Whether the continuous measurements in the study were normally distributed or not was examined by Kolmogorov-Smirnov $(n>50)$ and Skewness-Kurtosis. Tests and parametric tests were applied because the measurements were normally distributed. Descriptive statistics for continuous variables in the study; mean and standard deviation; and categorical variables were expressed as numbers (n) and percentages (\%). The "Independent T-test" was used to compare "continuous measures" according to Prednol Treatment Groups. Chi-square test was used to determine the relationship between Prednol Treatment Groups and categorical variables. Statistical significance level was taken as $5 \%$ in the calculations and SPSS (IBM SPSS for Windows, ver.25) statistical package program was used for analysis.

\section{Results}

One hundred and eleven female patients diagnosed with IGM were included in the study. 57 patients received local corticosteroid (LC) therapy, and 54 patients received oral corticosteroid (OC) therapy. Patients' mean age was $32.7 \pm 7.08$ in the LC and $36.04 \pm 8.42$ in the $O C(p=0.014)$. The localization of lesions was similar between two groups $(p=0.224)$. The main complaint at onset presented with all three signs of disease as a breast mass with pain and skin erythema in two groups $(p=0.112)$. Duration until treatment was $10.98 \pm 21.08$ weeks in LC and $4.17 \pm 2.08$ weeks in OC $(p=0.020)$. The reason for the high duration of complaints in the LC group was the receipt of unsuccessful treatment, such as antibiotics, steroids, or other medications, or they are having undergone surgeries such as abscess drainage 
and lumpectomy in other hospitals. At the beginning, we performed tru-cut biopsy and started treatment early. The mean number of children was $2.62 \pm 1.58$ in the LC and $3.28 \pm 2.10$ in the $O C(p=0.066)$. Total breast-feeding time was $41.52 \pm 30.47$ months in the LC and $48.41 \pm 30.78$ months in the OC $(p=0.245)$. Smoking ratio was similar between the two groups $(p=0.248)$. Patients and their clinical characteristics are shown in Table 1.

Table 1

Patients and their clinical characteristics

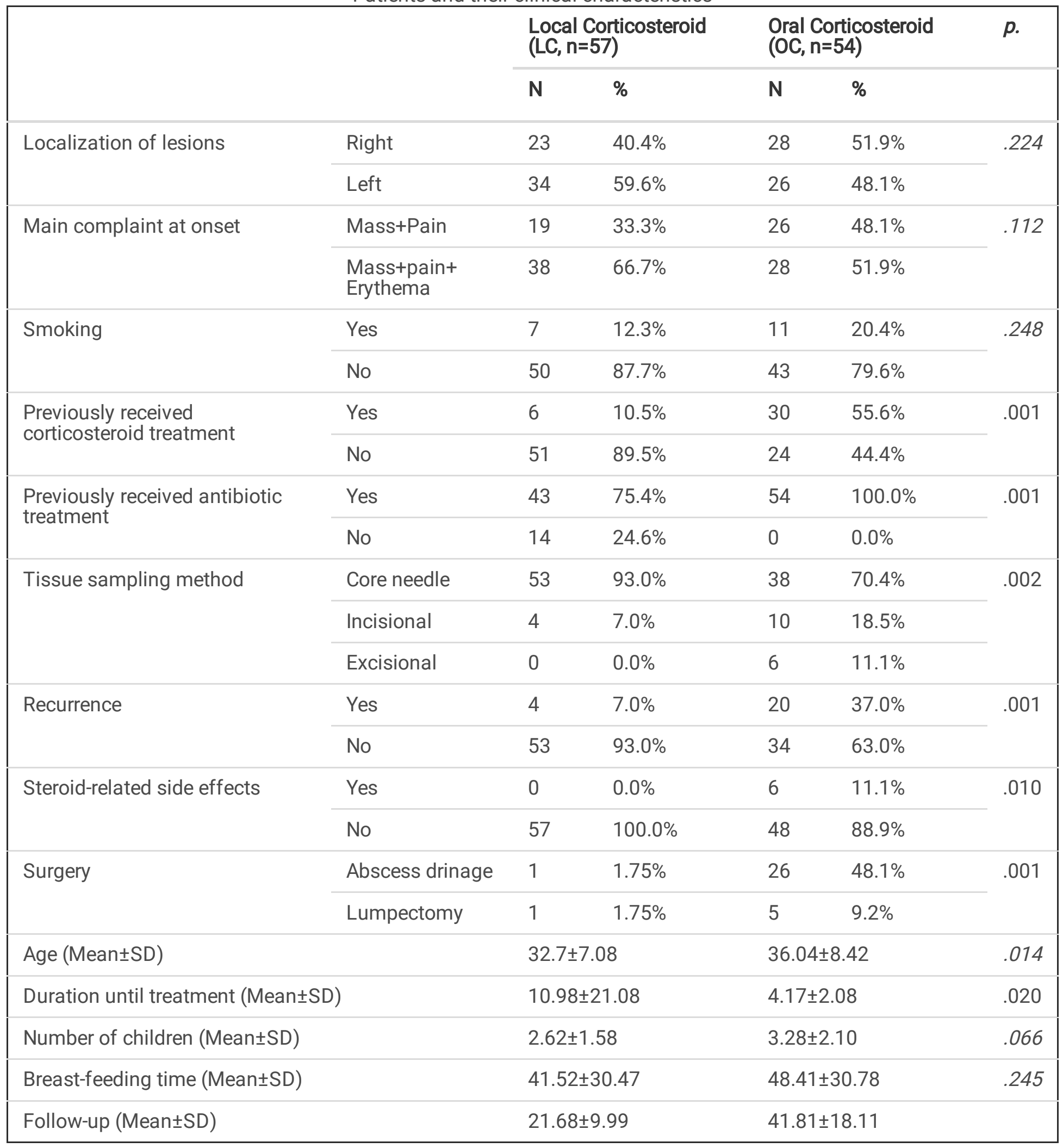


While 10.5\% (6 patients) of the patients in the LC group had previously received corticosteroid treatment, $55.6 \%$ (30 patients) in the OC group had previously received corticosteroid treatment ( $p=0.001)$. While $75.4 \%$ (43 patients) of the patients in the LC group had received antibiotic treatment, 100\% (54 patients) in the OC group had received antibiotic treatment initially $(p=0.001)$. This is due to the low response rate to oral steroids and starting antibiotics without a definitive diagnosis, making second and third doses necessary. Histopathological diagnosis was made by tru-cut biopsy in 93\% (53 patients) of the patients in the LC group, while the diagnosis was made by tru-cut biopsy in $70.4 \%$ (38 patients) in the OC group ( $p=0.002$ ). In addition, 18.5\% (10 patients) in the OC group were treated with incisional biopsy and 11.1\% (6 patients) with excisional biopsy (Table 1). The reason for the high rate of tru-cut biopsy in the LC group was that the diagnosis and treatment of these patients were made in our center, which is a university hospital.

In the LC group, two patients with a complete response after the first course of treatment received a second course of treatment due to recurrence. Similarly, a third course of treatment was given to two patients in the LC group who developed recurrence after the second course of treatment. In the LC group, lumpectomy was performed in one patient who did not achieve a complete response after the second course of treatment. We performed oncoplastic surgery on our own patients and their breast appearance was not so bad. The patient in Fig. 2 had surgery with the bad cosmetic result of surgery in another center. In the OC group, lumpectomy was performed in five patients who did not achieve a complete response. While there were no steroid-related side effects in the LC group, $11.1 \%$ (6 patients) of the OC group had steroid-related side effects such as steroid-induced hirsutism, acne, weight gain, diffuse bone pain, and muscle weakness $(p=0.010)$. These patients used steroids and these side effects were of course steroid related. These patients were also consulted to the endocrine clinic and followed up with them. The follow-up period for the patients was $21.68 \pm 9.99$ in the LC group and $41.81 \pm 18.11$ in the OC group. (Table 1).

While $7 \%$ (4 patients) of the patients in the LC group had recurrence, 37\% (20 patients) in the OC group had recurrence $(p=0.001)$. Surgery was performed in $3.5 \%$ (2 patients) in the LC group, while surgery was performed in $57.3 \%(31$ patients) in the OC group ( $\mathrm{p}=0.001)$. Abscess drainage was performed in $1.75 \%$ (1 patient), and lumpectomy was performed in $1.75 \%$ (1 patient) in the LC group, whereas abscess drainage was performed in $48.1 \%$ ( 26 patients) and lumpectomy in $9.2 \%$ (5 patients) in the OC group. The reason for the high number of surgical interventions in the OC group is the lower response rates to corticosteroid treatment and different treatment protocols (antibiotic, low dose oral corticosteroid) are used by other centers (Table 1).

In the first course of treatment, $28.1 \%$ in the LC group (14 partial response, 2 no response) did not achieve complete response, but $70.4 \%$ did not achieve complete response in the OC group (25 partial response, 12 no response and 1 worsening disease) $(p=0.001)$. In the second course of treatment, $12.3 \%$ (7 partial response) in the LC group and $22.2 \%$ in the OC group (10 partial response, 2 no response) did not achieve complete response $(p=0.058)$. In the third course of treatment, $1.75 \%$ (1 partial response) in the LC groupand 13.0\% in OC group (5 partial response, 2 no response) did not achieve complete response $(p=0.004)$ (Table 2$)$. 
Table 2

Response rates of patients in treatment arms

\begin{tabular}{|lllllll|}
\hline & \multicolumn{2}{l}{ 1st administration } & \multicolumn{2}{l|}{ 2nd administration } & \multicolumn{2}{l|}{ 3rd administration } \\
\cline { 2 - 5 } & $\begin{array}{l}\text { Local } \\
\text { Corticosteroid } \\
(\mathbf{n}=57)\end{array}$ & $\begin{array}{l}\text { Oral } \\
\text { Corticosteroid } \\
(\mathbf{n}=54)\end{array}$ & $\begin{array}{l}\text { Local } \\
\text { Corticosteroid } \\
(\mathbf{n}=18)\end{array}$ & $\begin{array}{l}\text { Oral } \\
\text { Corticosteroid } \\
(\mathbf{n}=37)\end{array}$ & $\begin{array}{l}\text { Local } \\
\text { Corticosteroid } \\
(\mathbf{n}=7)\end{array}$ & $\begin{array}{l}\text { Oral } \\
\text { Corticosteroid } \\
(\mathbf{n}=13)\end{array}$ \\
\hline $\begin{array}{l}\text { Complete } \\
\text { response }\end{array}$ & $41(71.9 \%)$ & $16(29.6 \%)$ & $11(19.2 \%)$ & $25(46.3 \%)$ & $6(10.5 \%)$ & $6(11.1 \%)$ \\
\hline $\begin{array}{l}\text { Partial } \\
\text { response }\end{array}$ & $14(24.6 \%)$ & $25(46.3 \%)$ & $7(12.3 \%)$ & $10(18.5 \%)$ & $1(1.75 \%)$ & $5(9.3 \%)$ \\
$\begin{array}{l}\text { No } \\
\text { response }\end{array}$ & $2(3.5 \%)$ & $12(22.2 \%)$ & 0 & $2(3.7 \%)$ & 0 & $2(3.7 \%)$ \\
\hline $\begin{array}{l}\text { Worsening } \\
\text { disease }\end{array}$ & 0 & $1(1.9 \%)$ & 0 & 0 & 0 & 0 \\
\hline
\end{tabular}

When patients with complete and partial response were pooled in a single group to determine the rate of "responders," $96.5 \%$ (55 patients) in the LC group and $75.9 \%$ (41 patients) in the OC group were responders after the first course of treatment ( $\mathrm{p}=0.001) ; 91.1 \%$ (52 patients) in the LC group and $75.9 \%$ (41 patients) in the OC group were complete responders after the second course of treatment; and $98.2 \%$ (56 patients) in the LC group and $87.0 \%$ (47 patients) in the OC group were complete responders after the third course of treatment $(p=0.003)$, (Figure $3 a, b$ and $4 a, b$ and $5 a, b)$. The fourth dose of treatment was administered to one patient who was stable and then continued with partial responses in the LC group (Table 2).

Accordingly, previous steroid use was significantly lower in the LC group (10.5\%) compared to OC group (55.6\%) $(p=0.001)$. Previous antibiotic use was significantly lower in the LC group $(75.4 \%)$ compared to the OC group $(100 \%)$ $(p=0.001)$. Treatment response rate was significantly higher in the LC group than in the OC group $(p=0.001)$. The recurrence rate was significantly lower in the LC group (7\%) compared to the OC group (37\%) $(p=0.001)$. Steroid-related side effects were lower in the LC group (none) compared to the OC group $(11.1 \%)(p=0.010)$. The need for surgical treatment was significantly lower in the LC group (3.5\%) compared to the OC group (57.3\%) $(p=0.001)$.

\section{Discussion}

Since Kessler and Wolloch defined IGM in 1972, despite time and developing technology and treatment modalities, uncertainty in the physiopathology of IGM and debates on its treatment continue [2,3]. Although autoimmunity is the cause most supported by the literature, various etiological factors such as various hormonal disorders (hyperprolactinemia, etc.), oral contraceptive use, autoimmune factors, microorganisms, smoking, and alpha 1antitrypsin deficiency have been blamed [8-10]. The fact that the physiopathology of IGM is not well known has hampered effective treatment. [11]. In addition to surgical and non-surgical treatment modalities, the use of local steroids has recently been discussed in the literature. In this study, we aimed to discuss cases with IGM treated with local and systemic steroids in light of current literature.

The rate of smoking and oral contraceptive use, which are etiological factors, was $0-23.7 \%$ and $15.3 \%-52.6 \%$, respectively [3,12-15]. In our study, while smoking was $12.3 \%$ in the LC group, it was $20.4 \%$ in the OC group. There was no history of oral contraceptive use in either group of our patients. In this context, the effects of smoking and oral contraceptive use on the pathophysiology of the disease are questionable. 
Although most patients receive antibiotic treatment before the definitive diagnosis because of diagnostic uncertainty, routine antibiotic use is not recommended given thats IGM is a sterile condition $[3,16]$. Oral steroid and antibiotic use rates were very high in the $\mathrm{OC}$ group. The lack of high treatment responses has led to the reuse of these medications. High doses and long-term use of these medications cause some complications and increase the cost of treatment. The fact that these were lower in the LC group (steroid use; $10.5 \%$, antibiotic use; $75.4 \%$ ) indicates the effectiveness of the local steroid treatment and a more effective professional approach to the disease. Similarly, the tru-cut biopsy rates are high in the LC group.

Although one of the most used methods in the treatment of IGM is steroid therapy and surgery, there are many options such as follow-up, antibiotics, non-steroidal anti-inflammatory drugs, colchicine, methotrexate, azathioprine, imuran, and mycophenolate mofetil. And there is a current study about local steroid use $[3,11-13,15,17,18]$. There are opinions that wide local excision is the most ideal treatment. However, surgical excision may have a high recurrence rate, poor wound healing, fistulization, and cosmetic problems [19]. In recent years, medical treatments have been more preferred in the treatment of IGM [15]. Corticosteroids have been defined as first-line therapy because of their positive results [12]. On the other hand, steroids are known to cause side effects that affect all systems such as blood sugar irregularity (steroid-induced diabetes mellitus), weight gain, Cushing's syndrome, and body weight gain, and as a result they require a change in treatment algorithm [11, 15, 20-22]. Topical corticosteroids are used in the treatment of many skin diseases, especially atopic dermatitis, due to their anti-inflammatory, vasoconstrictive, antiproliferative and immunosuppressive effects. While steroids can be easily absorbed through normal skin, their absorption at the site of inflammation is increased $[18,23]$.

Topical steroid use was first described in an IGM case by Altintoprak et al. in 2011. They saw a significant improvement in the patient's clinic after 5 weeks of treatment [18]. In their next studies, they treated 28 patients with only topical steroid for an average of 8.2 weeks (range 4-12 weeks) and noted that the long-term efficacy of treatment was more than 90\% [11]. After that Munot et al. applied local steroids to the breast lesion in 4 patients and noted clinical and radiological improvement in all patients [24]. In our previous study, in which we treated patients with uncomplicated IGM with local steroids, we showed that there was $93.5 \%$ improvement in the lesion in the first month after the first dose. The rate of patients with complete response after the third dose was $93.4 \%$ [3].

When we look at the treatment durations of different studies in the literature; Lai et al. [25] found that the duration of treatment in the observation treatment group was 14.5 months. Cetinkaya et al. [15] in their study, found that the duration of improvement in the observation group was 5.6 months (1.3-13.8) and 3.9 months (0.8-9.8) in the systemic steroid treatment group. Azizi et al. [12] described the recovery period of the observation group as 9 months in their study. By 15 months of treatment with MTX, 75\% achieved disease remission, and 13-15 (range 1-30) months were median duration of treatment [13]. In a study by Montazer et al. [26] improvement was seen after 12 months in $93.3 \%$ of patients in the group treated with high-dose steroids (50 mg daily), while $53.3 \%$ of patients in the low-dose steroid group were able to improve after 6 months [27]. In their study in which they performed steroid injection, Alper et al. showed that $89.3 \%$ of the patients had complete recovery in their mean follow-up of 11.8 (5-20) months. In our study, 98.2\% (56 patients) in the LC group and 87.0\% (47 patients) in the OC group were complete responders after three months $(p=0.001)$. When comparing our study with the literature, we see that the treatment time in the the LC group was significantly shorter. In other words, we see that we can reach a high cure rate in a shorter time.

Equally as difficult as treatment in patients with IGM is the recurrence of the disease. Although it varies according to the type of treatment in the literature, the recurrence rate in IGM is reported to be between $5-50 \%$. The recurrence rate in this study was $24.8 \%$ [12]. Montazer et al. [26] found no significant difference in the recurrence rate between the conservative and surgical treatment groups. Cetinkaya et al. [15] found that recurrence rates were $14.3 \%$ in the 
observation group, $42.9 \%$ in the systemic corticosteroid group, and $21.4 \%$ in the antibiotic group. Postolova et al. [13] found that recurrence rate was $50 \%$ after surgery. They found that $15.8 \%$ of patients had recurrence during their treatment with MTX. In our study, while 7\% (4 patients) of the patients in the LC group had recurrence, $37 \%$ (20 patients) in the OC group had recurrence $(p=0.001)$. The literature indicates that the recurrence rate was significantly lower according to the use of systemic.

The greatest restriction on the use of the steroid is undoubtedly its side effects. Cetinkaya et al. [15] reported in their study that the treatment was not successful in more than half of the patients they treated with steroids and that the patients had problems due to various side effects. In another study involving treatment with MTX, side effects were reported in $15.7 \%$ of patients [13]. Yin et al. [2] stated in their article that the side effects of topical, systemic, and combined steroid treatments were $2.4 \%, 38.2 \%$ and $30.3 \%$, respectively. Munot et al. [24] reported they had no side effects in any of the 4 patients they treated with steroid injection [27]. In a study in which they used local steroids, Alper et al. found that none of the 28 patients with IGM observed steroid-related side effects. In our previous study, which was multicentric, the rate of steroid-related side effects was $2.2 \%$ in the local steroid group, while it was $9.4 \%$ in the systemic steroid group [3]. In our study, there were no steroid-related side effects in the LC group and 11.1\% (6 patients) in the OC group.

Wide surgical excision may be required to prevent recurrence; however, it causes large defects in the breast, resulting in negative cosmetic outcomes and psychological burden [2]. Chirappapa et al. [28] reported in their cohort study that surgical resection offered no advantage over steroids in terms of healing time, and even $52 \%(13 / 25)$ of patients had wound complications. Shin et al. [29] reported that surgical resection may result in significant breast deformity, extensive scarring, and other complications. In our study, the patients' need for surgery was 3.5\% in the LC group and $59.3 \%$ in the OC group $(p=0.001)$.

\section{Conclusion}

Steroid injection is a treatment that is easy to administer and minimizes the risk of systemic side effects. It carries with it a proven efficacy, short response time, and low recurrence rate. Even in clinically severe cases, we believe that steroid injection might be used as monotherapy, or in combination with other therapies, to improve treatment efficacy and create a transition to less aggressive treatments. Prospective randomized studies investigating the effect of different doses of local steroid injections, long-term responses, and side effects should be carried out to aid in the development of future treatment algorithms for patients with IGM.

Each treatment modality has advantages and disadvantages. Considering the superiority of local steroid use to other treatments, it should be considered a primary treatment modality.

\section{Declarations}

\section{Acknowledgement}

Thanks so much to everyone who contributed from the beginning of the article to its publication.

\section{Statement of Ethics}

This study was approved by the local ethics committee of Van Yüzüncü Yıl University, Turkey. (Reg. No. 2021/06-03). Informed consent was not received duo to the retrospective registry nature of the study 
Declaration of conflicting interests

The authors declare that there are no potential conflicts of interest with respect to the research, authorship, and/or publication of this article.

\section{Funding Sources}

The authors received no financial support for the research, authorship, and/or publication of this article.

\section{Author Contribution}

O.T., N.T., S.E., I.C. and S.B.: contributed to drafting the original grant proposal and obtained funding. O.T., N.T., and S.E.: did the analyses. All authors contributed to the interpretation of the data. O.T., S.E., I.C., and S.B.: drafted the manuscript. All authors revised the manuscript and approved the final version to be published.

\section{References}

1. Kessler E, Wolloch Y. Granulomatous mastitis: a lesion clinically simulating carcinoma. Am J Clin Pathol. 1972;58(6): 642-646. doi:10.1093/ajcp/58.6.642.

2. Yin Y, Liu X, Meng Q, Han X, Zhang H, Lv Y. Idiopathic Granulomatous Mastitis: Etiology, Clinical Manifestation, Diagnosis and Treatment. J Invest Surg. 2021 Mar 10:1-12. doi: 10.1080/08941939.2021.1894516.

3. Toktas O, Konca C, Trabulus DC, Soyder A, Koksal H, Karanlik H, Kamali Polat A, Ozbas S, Yormaz S, Isik A, Sezgin E, Soran A. A Novel First-Line Treatment Alternative for Noncomplicated Idiopathic Granulomatous Mastitis: Combined İntralesional Steroid İnjection with Topical Steroid Administration. Breast Care (Basel). 2021 Apr;16(2):181-187. doi: 10.1159/000507951.

4. Haitz K, Ly A, Smith G. Idiopathic granulomatous mastitis. Cutis. 2019;103(1):38-42.

5. Baslaim M, Khayat $\mathrm{H}$, Al-Amoudi S. Idiopathic granulomatous mastitis: a heterogeneous disease with variable clinical presenta- tion. World J Surg. 2007;31(8):1677-1681. doi:10.1007/s00268-007-9116-1.

6. Ma X, Min X, Yao C. Different treatments for granulomatous lobular mastitis: a systematic review and metaanalysis. Breast Care (Basel). 2020;15(1):60-66. doi:10.1159/000501498.

7. Kaviani A, Noveiry B, Jamei K, Rabbani A. How to manage idiopathic granulomatous mastitis: suggestion of an algorithm. Breast J. 2014;20(1):110-112. doi:10.1111/tbj.12216.

8. Barreto DS, Sedgwick EL, Nagi CS, et al. Granulomatous mas- titis: etiology, imaging, pathology, treatment, and clinical find- ings. Breast Cancer Res Treat. 2018;171(3):527-534. doi:10.1007/s10549-018-4870-3.

9. Benson JR, Dumitru D. Idiopathic granulomatous mastitis: presentation, investigation and management. Future Oncol. 2016;12(11):1381-1394. doi:10.2217/fon-2015-0038.

10. Saydam M, Yilmaz KB, Sahin M, Yanik H, Akinci M, Yilmaz I, Balas S, Azili C, Gulcelik MA. New Findings on Autoimmune Etiology of Idiopathic Granulomatous Mastitis: Serum IL-17, IL-22 and IL-23 Levels of Patients. J Invest Surg. 2020 Feb 11:1-5. doi: 10.1080/08941939.2020.1725190.

11. Altintoprak F, Kivilcim T, Yalkin O, Uzunoglu Y, Kahyaoglu Z, Dilek ON. Topical Steroids Are Effective in the Treatment of Idiopathic Granulomatous Mastitis. World J Surg. 2015 Nov;39(11):2718-23. doi: 10.1007/s00268015-3147-9.

12. Azizi A, Prasath V, Canner J, Gharib M, Sadat Fattahi A, Naser Forghani M, Sajjadi S, Farhadi E, Vasigh M, Kaviani A, Omranipour R, Habibi M. Idiopathic granulomatous mastitis: Management and predictors of recurrence in 474 patients. Breast J. 2020 Jul;26(7):1358-1362. doi: 10.1111/tbj.13822. 
13. Postolova A, Troxell ML, Wapnir IL, Genovese MC. Methotrexate in the Treatment of Idiopathic Granulomatous Mastitis. J Rheumatol. 2020 Jun 1;47(6):924-927. doi: 10.3899/jrheum.181205.

14. Zhang X, Li Y, Zhou Y, Liu D, Chen L, Niu K, Sun Q, Huang H. A systematic surgical approach for the treatment of idiopathic granulomatous mastitis: a case series. Gland Surg. 2020 Apr;9(2):261-270. doi: 10.21037 /gs.2020.02.06.

15. Cetinkaya G, Kozan R, Emral AC, Tezel E. Granulomatous mastitis, watch and wait is a good option. Ir J Med Sci. 2020 Oct 20. doi: 10.1007/s11845-020-02406-0.

16. Dag A, Edizsoy A. Challenges in management of idiopathic granulomatous mastitis during the pandemic of COVID-19. Breast J. 2021 Jan;27(1):87-88. doi: 10.1111/tbj.14092.

17. Cetin K, Sıkar HE, Goret NE, Rona G, Barısık NO, Kucuk HF, Gulluoglu BM. Comparison of Topical, Systemic, and Combined Therapy with Steroids on Idiopathic Granulomatous Mastitis: A Prospective Randomized Study. World J Surg, 2019. 43(11): p. 2865-73

18. Altintoprak F. Topical steroids to treat granulomatous mastitis: a case report. Korean J Intern Med. 2011 Sep;26(3):356-9. doi: 10.3904/kjim.2011.26.3.356.

19. Gurleyik G, Aktekin A, Aker F et al (2012) Medical and surgical treatment of idiopathic granulomatous lobular mastitis: a benign inflammatory disease mimicking invasive carcinoma. J Breast Cancer 15(1):119-123. https://doi.org/10.4048/jbc.2012.15.1.119.

20. Chrousos GP (2001) Glucocorticoid therapy. In: Felig P, Froh- man L (eds) Endocrinology and metabolism, 4th edn. McGraw- Hill, Incorporated, New York, pp 609-612.

21. Raj N, Macmillan RD, Ellis IO et al (2004) Rheumatologists and breasts: immunosuppressive therapy for granulomatous mastitis. Rheumatology 43(8):1055-1056.

22. Wolfrum A, Kümmel S, Theuerkauf I et al (2018) Granulomatous mastitis: a therapeutic and diagnostic challenge. Breast Care (Basel) 13(6):413-418. https://doi.org/10.1159/000495

23. Buys LM. Treatment options for atopic dermatitis. Am Fam Physician 2007;75:523-528.

24. Munot K, Nicholson S, Birkett V. Granulomatous Mastitis e a novel method of treatment. Eur J Surg Oncol. 2012;38:461e462.

25. Lai EC, Chan WC, Ma TK et al () The role of conservative treatment in idiopathic granulomatous mastitis. Breast $J$ 2005;11(6):454-456. https://doi.org/10.1111/j.1075-122X.2005.00127.x

26. Montazer M, Dadashzadeh M, Moosavi Toomatari SE. Comparison of the Outcome of Low Dose and High-Dose Corticosteroid in the Treatment of Idiopathic Granulomatous Mastitis. Asian Pac J Cancer Prev. 2020 Apr 1;21(4):993-996. doi: 10.31557/APJCP.2020.21.4.993.

27. Alper F, Karadeniz E, Güven F, Yılmaz Çankaya B, Özden K, Akçay MN. The evaluation of the efficacy of local steroid administration in idiopathic granulomatous mastitis: The preliminary results. Breast J. 2020 Feb;26(2):309-311. doi: 10.1111/tbj.13588.

28. Chirappapha P, Thaweepworadej P, Supsamutchai C, Biadul N, Lertsithichai P. Idiopathic granulomatous mastitis: a retrospect- ive cohort study between 44 patients with different treatment modalities. Ann Med Surg. 2018;36:162-167. doi:10.1016/j.amsu. 2018.11.001.

29. Shin Y, Park S, Song Y, Son S, Choi Y. Is surgical excision necessary for the treatment of granulomatous lobular mastitis? BMC Womens Health. 2017;17(1):49. doi:10.1186/s12905-017-0412-0.

\section{Figures}




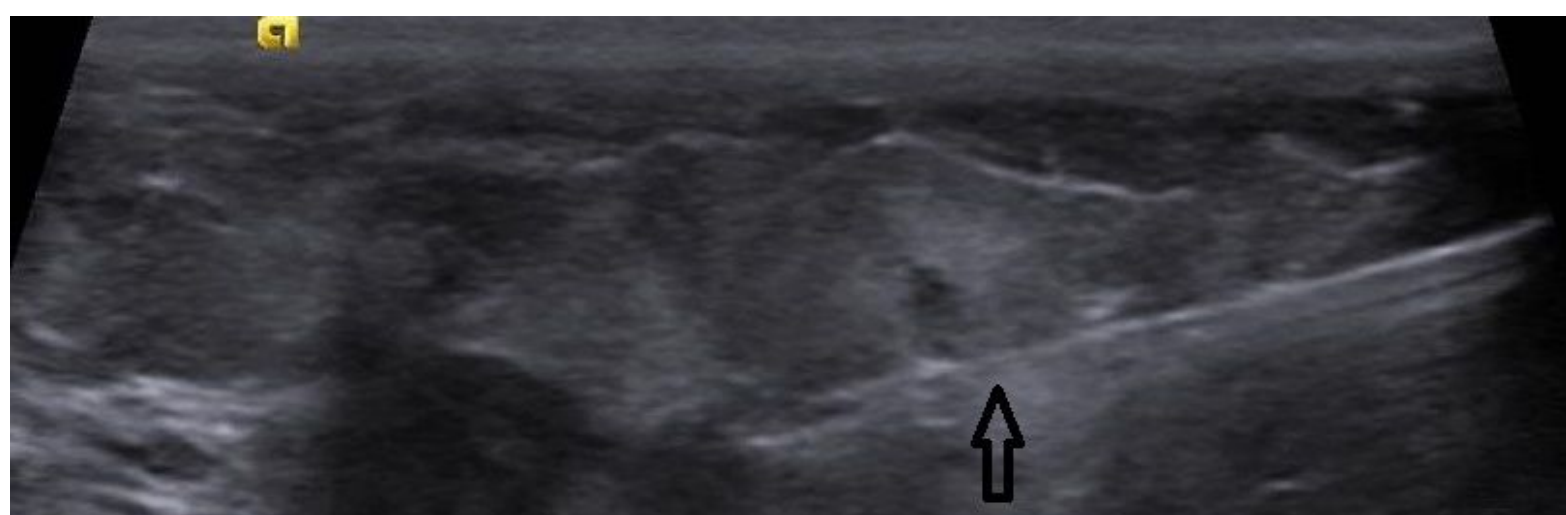

SIEMENS

14L5

Breast

General

MI: 1.0

$52 f p s$

2D-- $100 \%$

THI

$\mathrm{H} 11.00 \mathrm{MHz}$

20dB DR65

CTI 1

ASC 5

DTCE M

MapE/ST3

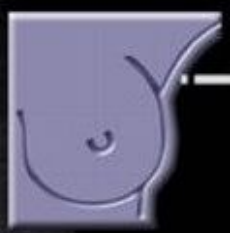

\section{Figure 1}

Ultrasound-guided steroid injection (Thin arrow is the needle in the center of IGM lesion). 


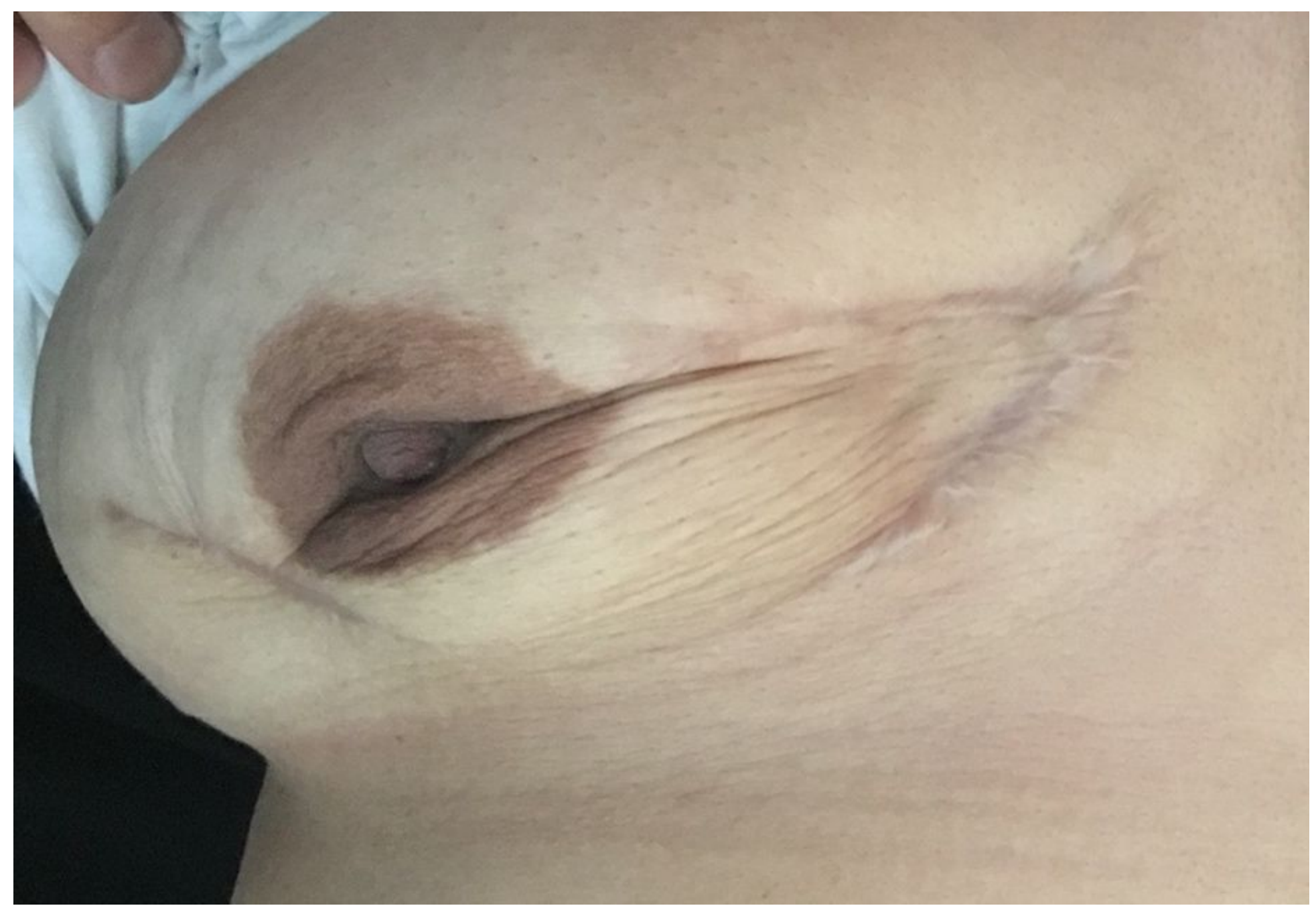

Figure 2

Poor cosmetic result after surgery. 


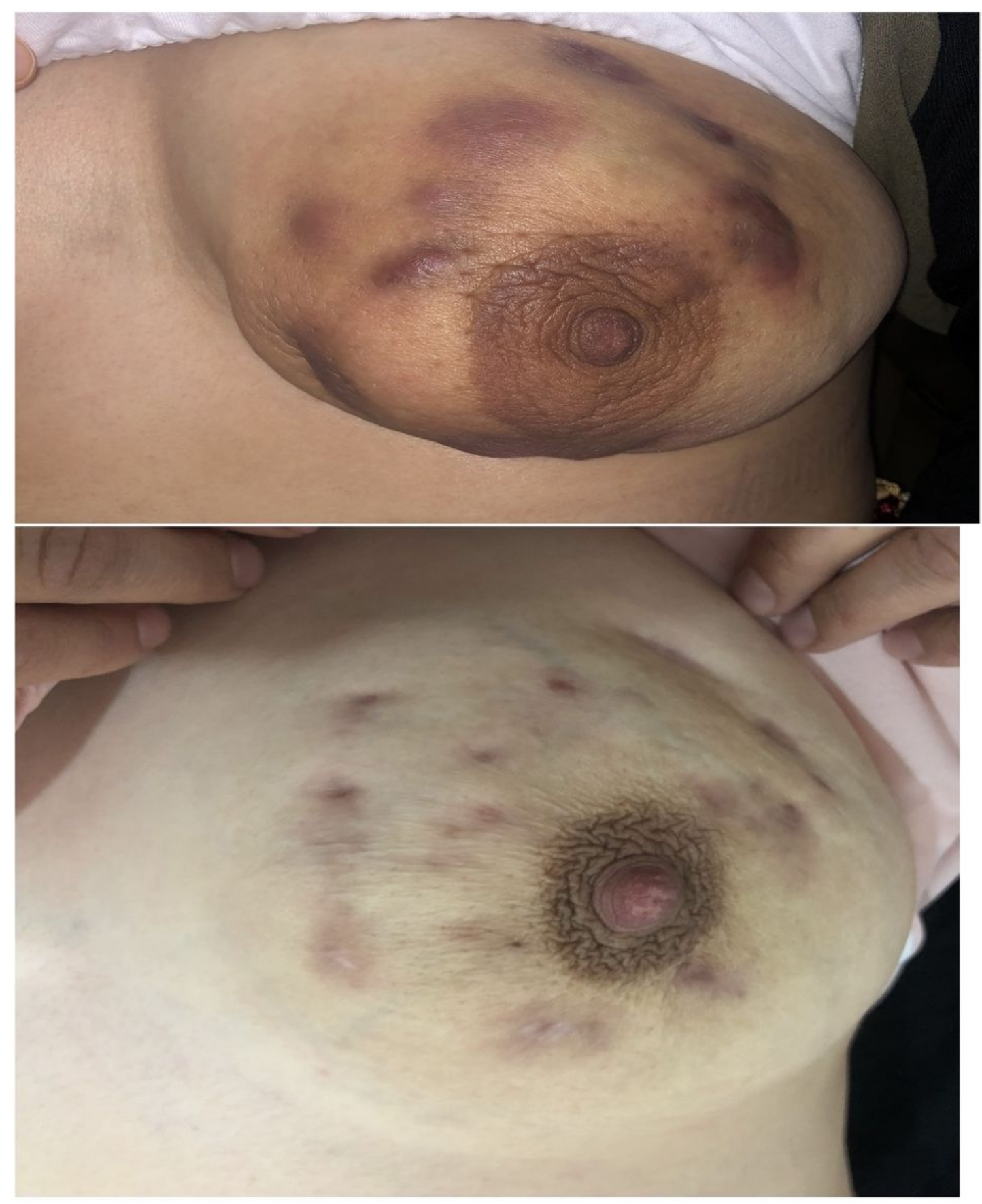

\section{Figure 3}

a/b: Follow-up of the patient who received steroid injection; a) Before treatment, b) After 3rd dose. 


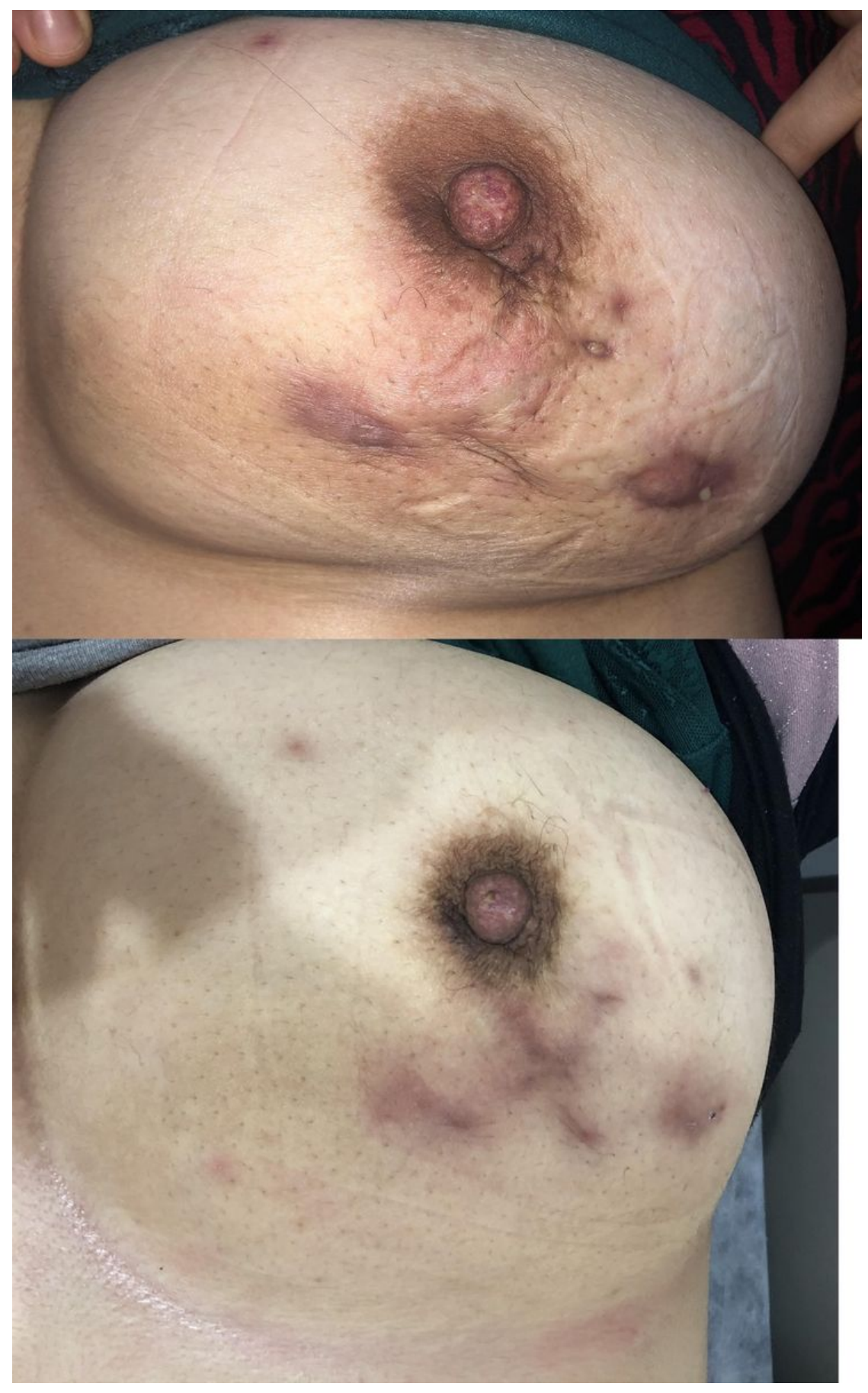

Figure 4

a/b: Follow-up of another patient who received steroid injection; a) Before treatment, b) After 2nd dose. 


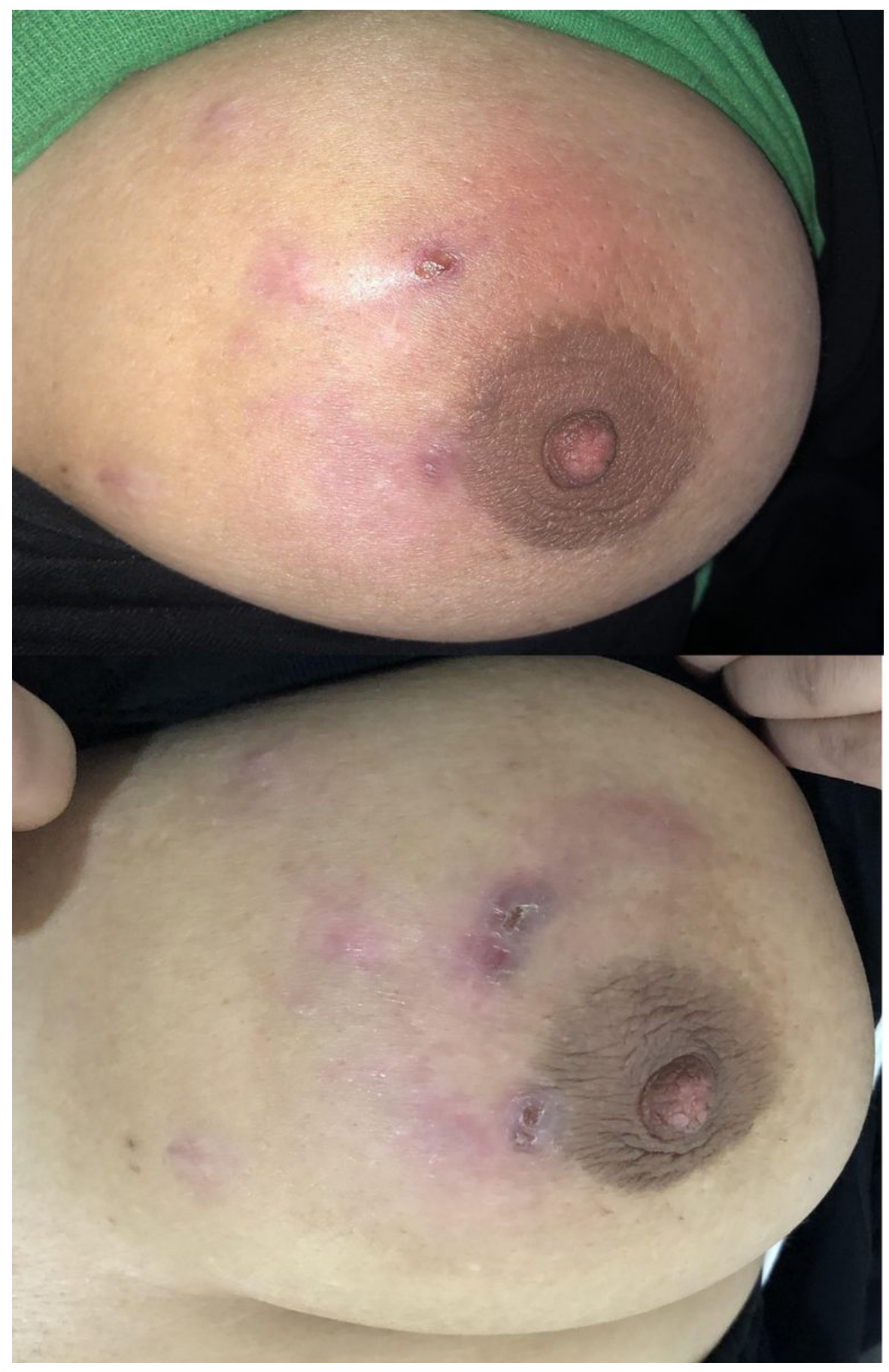

\section{Figure 5}

a/b: Follow-up of the patient who received oral steroid; a) Before treatment, b) After 3rd dose. 